\title{
Orientation control of $\mathrm{LiCoO}_{2}$ epitaxial thin films on metal substrates
}

Susumu Shiraki, ${ }^{\text {a* }}$ Yoshitaka Takagi, ${ }^{a}$ Ryota Shimizu, ${ }^{a}$ Tohru Suzuki, ${ }^{a}$ Masakazu Haruta, ${ }^{\mathrm{a}, 1}$ Yukio Sato, ${ }^{\mathrm{b}, 2}$ Yuichi Ikuhara, ${ }^{\mathrm{a}, \mathrm{b}, \mathrm{c}}$ and Taro Hitosugi ${ }^{\mathrm{a}}$

a Advanced Institute for Materials Research (AIMR), Tohoku University, 2-1-1 Katahira, Aoba, Sendai, Miyagi 980-8577, Japan

${ }^{\mathrm{b}}$ Institute of Engineering Innovation, The University of Tokyo, 2-11-16 Yayoi, Bunkyo, Tokyo 113-8656, Japan

c Nanostructures Research Laboratory, Japan Fine Ceramics Center, 2-4-1 Mutsuno, Atsuta, Nagoya 456-8587, Japan

*Corresponding author

E-mail: shiraki@wpi-aimr.tohoku.ac.jp

${ }^{1}$ Present address: Organization for Research Initiatives and Development, Doshisha University, 1-3 Tatara-Miyakodani, Kyotanabe, Kyoto, 610-0321, Japan.

${ }^{2}$ Present address: Department of Materials Science and Engineering, Kyushu University, 744 Motooka, Nishi-ku, Fukuoka, 819-0395, Japan. 


\begin{abstract}
We demonstrate the deposition of $\mathrm{LiCoO}_{2}(11-20)$ epitaxial thin films on chemically etched $\mathrm{Pt}(110)$ substrates, and compare their electrochemical properties with those of $\mathrm{LiCoO}_{2}(10-14)$ epitaxial thin films on sputter-annealed $\mathrm{Pt}(110)$ reconstructed substrates. Cyclic voltammogram measurements of $\mathrm{LiCoO}_{2}(11-20)$ epitaxial films exhibit stable charge and discharge operations, despite the application of a compressive strain on the films.
\end{abstract}

\title{
Keywords
}

Epitaxial films; Lithium ion batteries; $\mathrm{LiCoO}_{2} ;$ Crystal orientation 


\section{Introduction}

The fabrication of high-quality epitaxial thin films of Li-battery materials is one of the key issues in the investigation of the physical and chemical properties of battery operations and in the improvement of battery performance. Epitaxial growth techniques provide avenues for investigating interfaces in a controlled manner. The control of crystal orientation and area enables quantitative studies that cannot be performed using particle systems.

$\mathrm{LiCoO}_{2}(\mathrm{LCO})$ with a layered-rhombohedral $\alpha-\mathrm{NaFeO}_{2}$ structure is of particular interest in battery materials. It has been widely used as a cathode material for commercially available lithium batteries, and is a promising cathode material for all-solid-state lithium batteries [1]. Epitaxial thin films with controlled crystal orientations can be achieved by selecting the low-index planes of the substrate surface $[2,3,4]$. We have deposited flat $\operatorname{LCO}(0001)$ films (the notation of crystal planes is based on a hexagonal setting) on step-and-terrace $\mathrm{Al}_{2} \mathrm{O}_{3}(0001)$ substrates [5], and confirmed their high crystal quality by directly observing $\mathrm{Li}$ atoms using transmission electron microscopy (TEM) [6]. Although crystal-orientation control techniques have been established for insulating and electrochemically inactive substrates, few researchers have addressed crystal-orientation control on metal substrates and their impact on electrochemical properties.

Preparing epitaxial films on metal substrates allows us to investigate in detail the electrochemical properties of the films [7]. We have successfully controlled the crystal orientation of LCO on $\operatorname{Pt}(110)-(2 \times 1)$ reconstructed substrate surfaces, and reported on battery operations using liquid and solid electrolytes [8]. The reconstructed $\operatorname{Pt}(110)$ surface consists of alternately arrayed $\{111\}$ nanofacets, exhibiting what is called a 
"missing row" zigzag structure. On the reconstructed Pt(110) surface, we observed the growth of $\mathrm{LCO}(10-14)$ epitaxial films, which have $\mathrm{CoO}_{2}$ layers tilted by $52^{\circ}$ with respect to the surface normal. In the layered structure of $\mathrm{LCO}, \mathrm{Li}$ ions have a predominant two-dimensional diffusion along the $\mathrm{CoO}_{2}$ layers, and it is unlikely that the $\mathrm{Li}$ ions can penetrate through a $\mathrm{CoO}_{2}$ layer to an adjacent layer. This implies that the (10-14)- and (11-20)-oriented LCO epitaxial films are suitable for smooth Li-ion conduction along a direction perpendicular to the LCO films, in contrast to the (0001)-oriented case. Hence, a comparison of the electrochemical properties of LCO(11-20), (10-14), and (0001) films would illustrate the dependence of ion conductivity and electrochemical stability on orientation $[2,3,9]$. However, there has been no report on the growth of $\operatorname{LCO}(11-20)$ epitaxial films on metal surfaces. Furthermore, the electrochemical properties of $\operatorname{LCO}(11-20)$ on metal substrates have not been reported on.

In this study, we investigate the effects of substrate surface treatments on the growth of LCO films and report on the epitaxial growth of $\operatorname{LCO}(11-20)$ films. We deposited LCO thin films on $\operatorname{Pt}(110)$ substrates etched with aqua regia, and found that the substrate surface treatment has a significant influence on the growth orientation of LCO films. Furthermore, the films exhibited different charge/discharge characteristics depending on the orientation, that is, the $\operatorname{LCO}(11-20)$ and (10-14) orientations.

\section{Experimental}

We employed Pt(110) single crystals as substrates, and prepared the substrate surfaces using two methods. One involved a $\operatorname{Pt}(110)$ surface etched with aqua regia for $5 \mathrm{~min}$. The other involved a reconstructed $\operatorname{Pt}(110)-2 \times 1$ surface prepared by extensive 
sputtering-annealing cycles in ultrahigh vacuum [10]. Thin films of LCO were deposited on the $\mathrm{Pt}(110)$ substrates via pulsed laser deposition [5,8]. A Li-rich target, $\mathrm{Li}_{1.2} \mathrm{CoO}_{2}$, was used to compensate for the loss of $\mathrm{Li}$ during deposition. $\mathrm{A} \mathrm{KrF}$ excimer laser (wavelength: $248 \mathrm{~nm}$ ) was used to irradiate the target at a repetition rate of $5 \mathrm{~Hz}$. The fluence at the target was $1.0 \mathrm{~J} / \mathrm{cm}^{2}$. During the deposition process, the partial pressure of oxygen was $1 \times 10^{-6}$ Torr, and the substrate was kept at room temperature.

The as-grown samples were epitaxial films, but $\mathrm{Li}$ and $\mathrm{Co}$ cations were randomly distributed along the c-axis, as discussed by Wang et al. [11] and Bouwman et al. [12]. Hence, the as-grown films were subsequently annealed at $650{ }^{\circ} \mathrm{C}$ in air to obtain a layered-rhombohedral $\alpha-\mathrm{NaFeO}_{2} \mathrm{LCO}$ phase [5]. The LCO films were typically $100-\mathrm{nm}$ thick. The crystal structures of the films were characterized using X-ray diffraction (XRD) (D8 Discover, Bruker) and TEM (JEM-2100HC, JEOL Ltd.) [6]. Cyclic voltammetry $(\mathrm{CV})$ measurements were conducted using $1 \mathrm{M}$ of $\mathrm{LiClO}_{4}$ in ethylene carbonate/diethyl carbonate (1:1 by volume) as the electrolyte, with Li metal foils as anodes. The $\mathrm{CV}$ measurements were made at a scan rate of $0.1 \mathrm{mV} / \mathrm{s}$.

\section{Results and discussions}

First, we discuss the growth of LCO on the Pt(110) substrates chemically etched with aqua regia. The out-of-plane XRD pattern of the LCO film grown on the chemically etched Pt(110) substrate shows an LCO 11-20 peak (Fig. 1(a)). The in-plane XRD pattern (Fig. 1(b)) shows LCO 0003, 0006, and 0009 peaks, in addition to a Pt 111 peak. The $\phi$-scans obtained at $\psi=90^{\circ}\left(\psi=0^{\circ}\right.$ is the surface normal) show that the LCO 0003 and Pt 111 peaks appear at the same $\phi$ angles. These results indicate that (11-20)-oriented epitaxial films were grown on the etched $\operatorname{Pt}(110)$ substrates, and the 
in-plane epitaxial relationship can be described as $[0003]_{\mathrm{LCO}}(11-20)_{\mathrm{LCO}} / /[111]_{\mathrm{Pt}}(110)_{\mathrm{Pt}}$. In this orientation, the c-axis and the $\mathrm{CoO}_{2}$ layers in LCO are parallel and perpendicular to the $\operatorname{Pt}(110)$ surface, respectively, as illustrated in the inset of Fig. 1(a).

In addition to the $\operatorname{LCO}(11-20)$ phase, we found a minor secondary phase with a different crystal orientation. As shown in Fig. 2(a), a $\theta-2 \theta$ scan obtained at $\psi=35^{\circ}$ exhibits LCO 0003 and 0006 peaks in addition to a Pt 111 peak. This result indicates the epitaxial growth of $\operatorname{LCO}(01-18)$, which has a (0001) plane tilted by $35^{\circ}$ with respect to the $\mathrm{Pt}(110)$ surface normal (inset of Fig. 2(a)). An identical secondary phase was also observed on the large $\{111\}$ nanofacets formed on the reconstructed $\operatorname{Pt}(110) 2 \times 1$ surface [8], but the volume fraction was much smaller than that on the chemically etched substrate, as confirmed from the XRD peak intensity. Hence we speculate that, owing to the surface roughness of the etched $\mathrm{Pt}(110)$ substrate, the (111) facet of Pt is exposed on the topmost surface. Considering the epitaxial relationship between the $[0003]_{\text {LCO }}$ and (111) $)_{\mathrm{Pt}}$, we expect the growth of $\operatorname{LCO}(0001)$ film on this (111) facet. Indeed, exposure of the $\operatorname{Pt}(111)$ facets on the chemically etched $\mathrm{Pt}(110)$ substrate surface is suggested from surface morphology measurements.

An atomic force microscopy (AFM) image of an etched Pt surface shows large surface roughness of $R_{\mathrm{a}}=29.1 \mathrm{~nm}$ (Fig. 3(a)), which is in contrast to the smaller value observed on the reconstructed $\operatorname{Pt}(110)$ surface $\left(R_{\mathrm{a}} \approx 0.93 \mathrm{~nm}\right)$ (Fig. 3(b)). The surface roughness increases with increasing etching time. When the surface roughness $R_{\mathrm{a}}$ was $18.3 \mathrm{~nm}$ prepared by chemically-etching for $3 \mathrm{~min}$, a mixture of $\operatorname{LCO}(11-20)$ and (10-14) phases was grown (not shown here). This implies that the orientation of LCO films depends on the surface roughness of Pt substrate.

Cross-sectional TEM observation reveals microscopic feature of the LCO films (Fig. 
3(c)). The film surface is rough, which agrees with Fig. 3(a) and the thickness is approximately $100 \mathrm{~nm}$ as we aimed. The thickness is nearly constant throughout the film. We noticed that film surface morphology is quite similar to substrate surface one. This suggests that substrate surface roughness is a cause of film surface roughness. An electron diffraction pattern taken from the interface region confirms that the LCO film epitaxially grows on the Pt substrate (Fig. 3(d)). The patterns include two sets of hexagonal patterns: One is from Pt observed along [111] direction and the other one is from LCO observed along [0001] direction. This shows that [111] of Pt is parallel to [0001] of LCO in the in-plane direction, which agrees with the in-plane XRD profile in Fig. 1(b). On the other hand, top spots in both of the hexagonal patterns indicate (110) of Pt and (11-20) of LCO. This is again consistent with the out-of-plane XRD profile in Fig. 1(a).

Finally, we examined the electrochemical properties of the epitaxial $\operatorname{LCO}(11-20)$ film and compared them with those of the LCO(10-14) film. Figure 4(a) shows the CV curves of an electrochemical cell consisting of the $\operatorname{LCO}(11-20)$ film, a liquid electrolyte, and a Li anode. The peaks observed in the charge and discharge cycles correspond to the extraction and insertion of $\mathrm{Li}$ ions in $\mathrm{LCO}$, respectively. The appearance of those peaks at $3.94(\mathrm{C} 1), 3.97\left(\mathrm{C}^{\prime}\right), 4.08(\mathrm{C} 2)$, and $4.18 \mathrm{~V}(\mathrm{C} 3)$ in the charging process (and at 3.89 (D1'), 4.06 (D2), and $4.16 \mathrm{~V}$ (D3) in the discharging process) is a typical feature observed in the CV curves of polycrystalline bulks and films $[13,14,15,16]$. The C1' peak is also observed in Fig. 4(b), and the peak current at $3.85 \mathrm{~V}$ (D1') in the discharge process decreases to about half of $\mathrm{C}^{\prime}$ ' in the charging one. This implies that the D1' peak in Fig.4(a) is much smaller than D1, resulting in the double (C1 and $\left.\mathrm{C} 1{ }^{\prime}\right)$ and single peaks (D1) in the charging and discharging processes, respectively. Furthermore, 
we confirmed stable charging and discharging processes in the $\operatorname{LCO}(11-20)$ film, where the characteristics did not degrade even after the third cycle. In contrast, the LCO(10-14) film exhibits apparent degradation on cycling (Fig. 4(b)), which was also observed in the $\mathrm{LCO}(10-14)$ film on a $\mathrm{Au}(110)$ substrate [8]. Hirayama et al. carried out in-plane XRD of $\mathrm{LiMn}_{2} \mathrm{O}_{4}$ epitaxial films using synchrotron x-rays, and found small lattice change during charge-discharge operations. They concluded that the lattice change in the epitaxial films grown on single-crystal substrates was restricted during battery operations [17]. The same behaviors were also observed for epitaxial films, such as $\mathrm{Li}_{2} \mathrm{RuO}_{3}$ [18] and $\mathrm{LiNi}_{0.5} \mathrm{Mn}_{0.5} \mathrm{O}_{2}$ [19]. Furthermore, Ichitsubo et al. reported that the discharge capacity of Sn electrodes was only about $1 \%$ of the ideal value of $\mathrm{Sn}$ when the samples are under mechanical strain [20]. In the case of LCO(11-20), therefore, it is reasonable to assume that c-lattice expansion and contraction is having an impact on charge/discharge cycles, and thus we expected that fast degradation would take place during $\mathrm{Li}$ insertion and extraction reactions [21]. When the film is in a charged state, the c-lattice should undergo a lattice expansion of $\sim 2 \%$ [22], as a result of $\mathrm{Li}$ extraction from the film. The volume change might be hindered in the $\operatorname{LCO}(11-20)$ films, as the c-axis in $\operatorname{LCO}(11-20)$ is parallel to the surface. The film, however, does not exhibit a significant difference between the second and third cycles, strongly suggesting that the film possesses good electrochemical properties under compressive strain [2,9]. The voltage dependence of c-lattice in the epitaxial LCO films is still unknown. Further work of these systems is needed to show the effects of lattice strain in epitaxial films on the electrochemical properties.

\section{Conclusions}


We successfully fabricated epitaxial $\mathrm{LiCoO}_{2}(11-20)$ thin films on $\mathrm{Pt}(110)$ single-crystal substrates etched with aqua regia. The crystal orientation of the epitaxial films was tuned by controlling the surface morphology of the substrate. Furthermore, we confirmed stable and repeatable charge/discharge characteristics, even though the film is compressed.

\section{Acknowledgments}

This study was supported by the Japan Society for the Promotion of Science (JSPS) through its Funding Program for World-Leading Innovation R\&D on Science and Technology (FIRST Program), and the Advanced Low Carbon Technology Research and Development Program (ALCA) of the Japan Science and Technology Agency (JST). The authors also acknowledge the support of Toyota Corporation. S. S. acknowledges a Grant-in-Aid for Scientific Research (No. 25390072) from the Ministry of Education, Culture, Sports, Science and Technology (MEXT), Japan. A part of TEM work was done in The Ultramicroscopy Research Center, Kyushu University. 


\section{Figure captions}

Fig. 1. (a) Out-of-plane and (b) in-plane XRD patterns of $\mathrm{LiCoO}_{2}$ thin films on a $\operatorname{Pt}(110)$ substrate etched with aqua regia. The inset of (a) shows the crystal alignment of $\mathrm{CoO}_{2}$ layers and Pt substrates. (c) The $\phi$-scans of LCO 0003 and Pt 111 diffractions obtained at $\psi=90^{\circ}$.

Fig. 2. XRD patterns of LCO thin films on a $\mathrm{Pt}(110)$ substrate etched with aqua regia. The inset shows the crystal alignment of $\mathrm{CoO}_{2}$ layers and the Pt substrate. (a) A $\theta-2 \theta$ scan obtained at $\psi=35^{\circ}$. (b) The $\phi$-scans of LCO 0003 and Pt 111 diffractions obtained at $\psi=35^{\circ}$.

Fig. 3. AFM images of (a) etched and (b) reconstructed $\operatorname{Pt}(110)$ surfaces. The insets show photos of the $\mathrm{Pt}(110)$ substrates after the surface treatments. The chemically etched $\operatorname{Pt}(110)$ surface becomes lusterless after the etching treatments. (c) A TEM image of LCO on etched $\mathrm{Pt}(110)$. (d) An electron diffraction pattern taken from the region including LCO and Pt substrate. Electron incident axis is parallel to [111] of Pt substrate and [0001] and LCO. Sets of white and yellow arrows indicate the diffraction spots from Pt and LCO, respectively.

Fig. 4. Cyclic voltammograms of $\mathrm{LiCoO}_{2} / \mathrm{Pt}(110)$ : (a) $\mathrm{LCO}(11-20)$ film on etched $\operatorname{Pt}(110)$ and (b) LCO(10-14) film on reconstructed $\operatorname{Pt}(110)$. 


\section{References}

1 US Department of Energy, "Basic research needs for electrical energy storage", Report of the Basic Sciences Workshop on Electrical Energy Storage (2007).

2 M. Hirayama, N. Sonoyama, T. Abe, M. Minoura, M. Ito, D. Mori, A. Yamada, R. Kanno, T. Terashima, M. Takano, K. Tamura, J. Mizuki, Characterization of electrode/electrolyte interface for lithium batteries using in situ synchrotron X-ray reflectometry - A new experimental technique for $\mathrm{LiCoO}_{2}$ model electrode, J. Power Sources 168 (2007) 493.

3 D. Takamatsu, Y. Koyama, Y. Orikasa, S. Mori, T. Nakatsutsumi, T. Hirano, H. Tanida, H. Arai, Y. Uchimoto, Z. Ogumi, Electrochemical and spectroscopic characterization of $\mathrm{LiCoO}_{2}$ thin-film as model electrode, J. Electrochem. Soc. 161 (2014) A1447.

4 K. Nishio, T. Ohnishi, K. Akatsuka, K. Takada, Crystal orientation of epitaxial $\mathrm{LiCoO}_{2}$ films grown on $\mathrm{SrTiO}_{3}$ substrates, J. Power Sources 247 (2014) 687.

5 T. Tsuruhama, T. Hitosugi, H. Oki, Y. Hirose, T. Hasegawa, Preparation of layered-rhombohedral $\mathrm{LiCoO}_{2}$ epitaxial thin films using pulsed laser deposition, Appl. Phys. Express 2 (2009) 085502.

6 R. Huang, T. Hitosugi, S. D. Findlay, C. A. J. Fisher, Y. H. Ikuhara, H. Moriwake, H. Oki, and Y. Ikuhara, Real-time direct observation of $\mathrm{Li}$ in $\mathrm{LiCoO}_{2}$ cathode material, Appl. Phys. Lett. 98 (2011) 051913.

7 M. Haruta, S. Shiraki, T. Suzuki, A. Kumatani, T. Ohsawa, Y. Takagi, R. Shimizu, T. Hitosugi, Negligible "negative space-charge layer effects" at oxide-electrolyte/electrode interfaces of thin-film batteries, Nano Lett. 15 (2015) 1498.

8 S. Shiraki, H. Oki, Y. Takagi, T. Suzuki, A. Kumatani, R. Shimizu, M. Haruta, T. Ohsawa, Y. Sato, Y. Ikuhara, T. Hitosugi, Fabrication of all-solid-state battery using epitaxial $\mathrm{LiCoO}_{2}$ thin films, J. Power Sources 267 (2014) 881.

9 P. J. Bouwman, B. A. Boukamp, H. J. M. Bouwmeester, P. H. L. Notten, Influence of diffusion plane orientation on electrochemical properties of thin film $\mathrm{LiCoO} 2$ electrodes, J. Electrochem. Soc. 149 (2002) A699.

10 S. Agnoli, T. Orzali, M. Sambi, A. Vittadini, M. Casarin, G. Granozzi, Ultrathin TiO2 films on $(1 \times 2)-\mathrm{Pt}(110)$ : a LEED, photoemission, STM, and theoretical 
investigation, J. Phys. Chem. C 112 (2008) 20038.

11 H. Wang, Y.-I. Jang, B. Huang, D.R. Sadoway, Y.-M. Chiang, TEM study of electrochemical cycling-induced damage and disorder in $\mathrm{LiCoO}_{2}$ cathodes for rechargeable lithium batteries, J. Electrochem. Soc. 146 (1999) 473.

12 J. Bouwman, B. A. Boukamp, H. J. M. Bouwmeester, H. J. Wondergem, P. H. L. Notten, Structural analysis of submicrometer $\mathrm{LiCoO}_{2}$ films, J. Electrochem. Soc. 148 (2001) A311.

13 I. Uchida, H. Sato, Preparation of binder-free, thin film $\mathrm{LiCoO}_{2}$ and its electrochemical responses in a propylene carbonate solution, J. Electrochem. Soc. 142 (1995) L139.

14 K. W. Kim, S. I. Woo, K. -H. Choi, K. -S. Han, Y. -J. Park, Microfabrication of $\mathrm{LiCoO}_{2}$ film using liquid source misted chemical deposition technique, Solid State Ionics 159 (2003) 25.

15 N. Kuwata, N. Iwagami, Y. Taniji, Y. Matsuda, J. Kawamura, Characterization of thin-film lithium batteries with stable thin-film $\mathrm{Li}_{3} \mathrm{PO}_{4}$ solid electrolytes fabricated by ArF excimer laser deposition, J. Electrochem. Soc. 157 (2010) A521.

16 Y. Iriyama, T. Kako, C. Yada, T. Abe, Z. Ogumi, Charge transfer reaction at the lithium phosphorus oxynitride glass electrolyte/lithium cobalt oxide thin film interface, Solid State Ionics 176 (2005) 2371.

17 M. Hirayama, H. Ido, K. Kim, W. Cho, K. Tamura, J. Mizuki, R. Kanno, Dynamic Structural Changes at $\mathrm{LiMn}_{2} \mathrm{O}_{4}$ /electrolyte interface during Lithium Battery Reaction, J. Am. Chem. Soc. 132 (2010) 15268

18 S. Tanimoto, M. Hirayama, K. Suzuki, K. Kim, Y. Zheng, K. Tamura, J. Mizuki, R. Kanno, Mechanistic studies on lithium interaction in a lithium-rich layered material using $\mathrm{Li}_{2} \mathrm{RuO}_{3}$ epitaxial film electrodes and in situ surface X-ray analysis, J. Mater. Chem. A 2 (2014) 17875.

19 K. Sakamoto, M. Hirayama, H. Konishi, N. Sonoyama, N. Dupré, D. Guyomard, K. Tamura, J. Mizuki, R. Kanno, Structural changes in surface and bulk $\mathrm{LiNi}_{0.5} \mathrm{Mn}_{0.5} \mathrm{O}_{2}$ during electrochemical reaction on epitaxial thin-film electrodes characterized by in situ X-ray scattering, Phys. Chem. Chem. Phys. 12 (2010) 3815.

20 T. Ichitsubo, S. Yukitani, K. Hirai, S. Yagi, T. Uda, E. Matsubara, 
Mechanical-energy influences to electrochemical phenomena in lithium-ion batteries, J. Mater. Chem. 21 (2011) 2701.

21 G. G. Amatucci, J. M. Tarascon, L. C. Klein, $\mathrm{CoO}_{2}$, the end member of the $\mathrm{Li}_{\mathrm{x}} \mathrm{CoO}_{2}$ solid solution, J. Electrochem. Soc. 43 (1996) 1114.

22 T. Ohzuku, A. Ueda, Solid-state redox reactions of $\mathrm{LiCoO}_{2}(\mathrm{R}-3 \mathrm{~m})$ for 4 volt secondary lithium cells, J. Electrochem Soc. 141 (1994) 2972. 

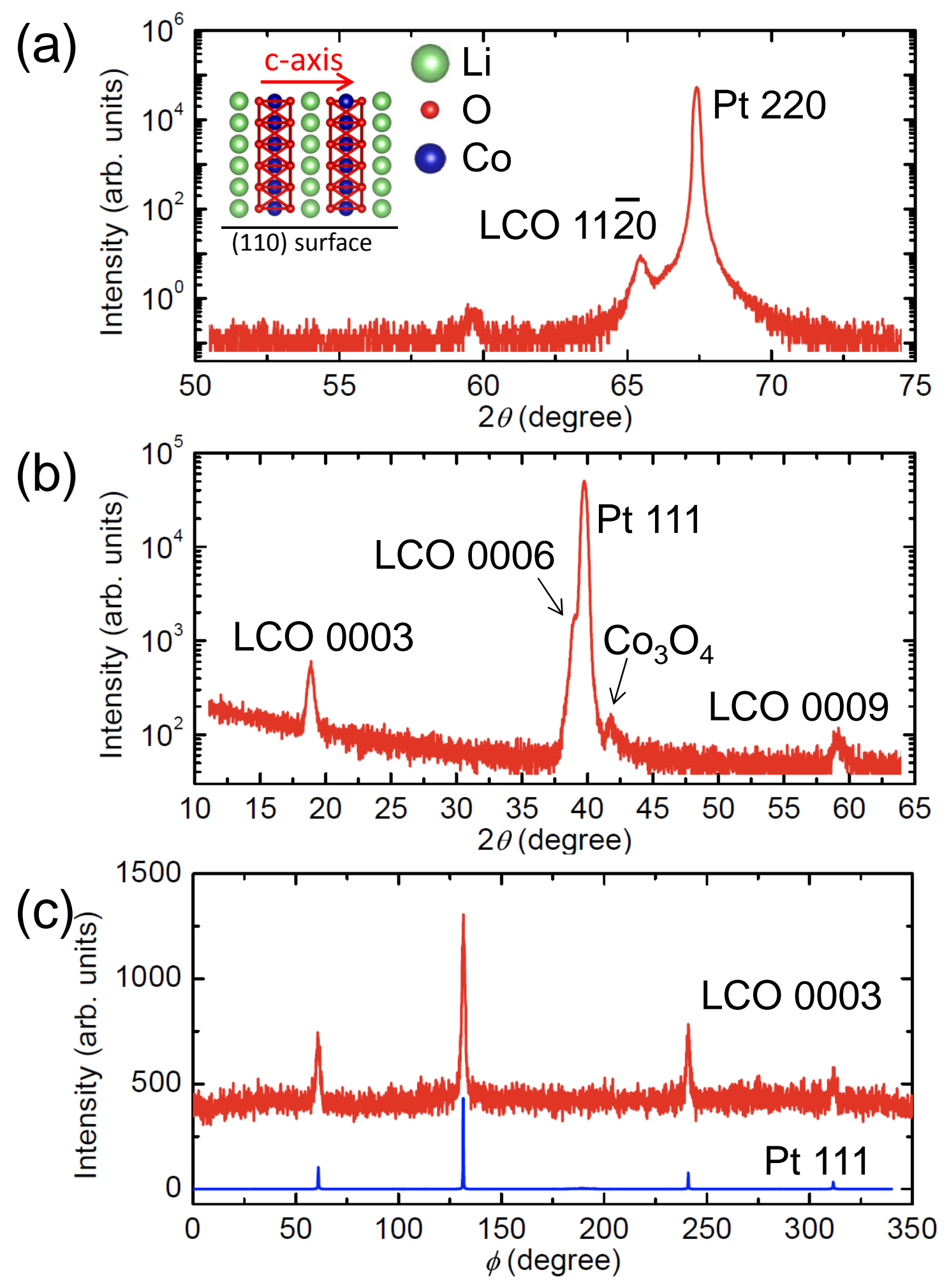

Fig. 1 XRD patterns of $\mathrm{LiCoO}_{2}$ thin films on a $\mathrm{Pt}(110)$ substrate etched with royal water. The inset shows a crystal alignment of $\mathrm{CoO}_{2}$ layers and Pt substrates. (a) Out-of-plane (b) In-plane (c) $\phi$-scan of $\mathrm{LiCoO}_{2} 0003$ and Pt 111 diffractions obtained at $\psi=$ $90^{\circ}$. 

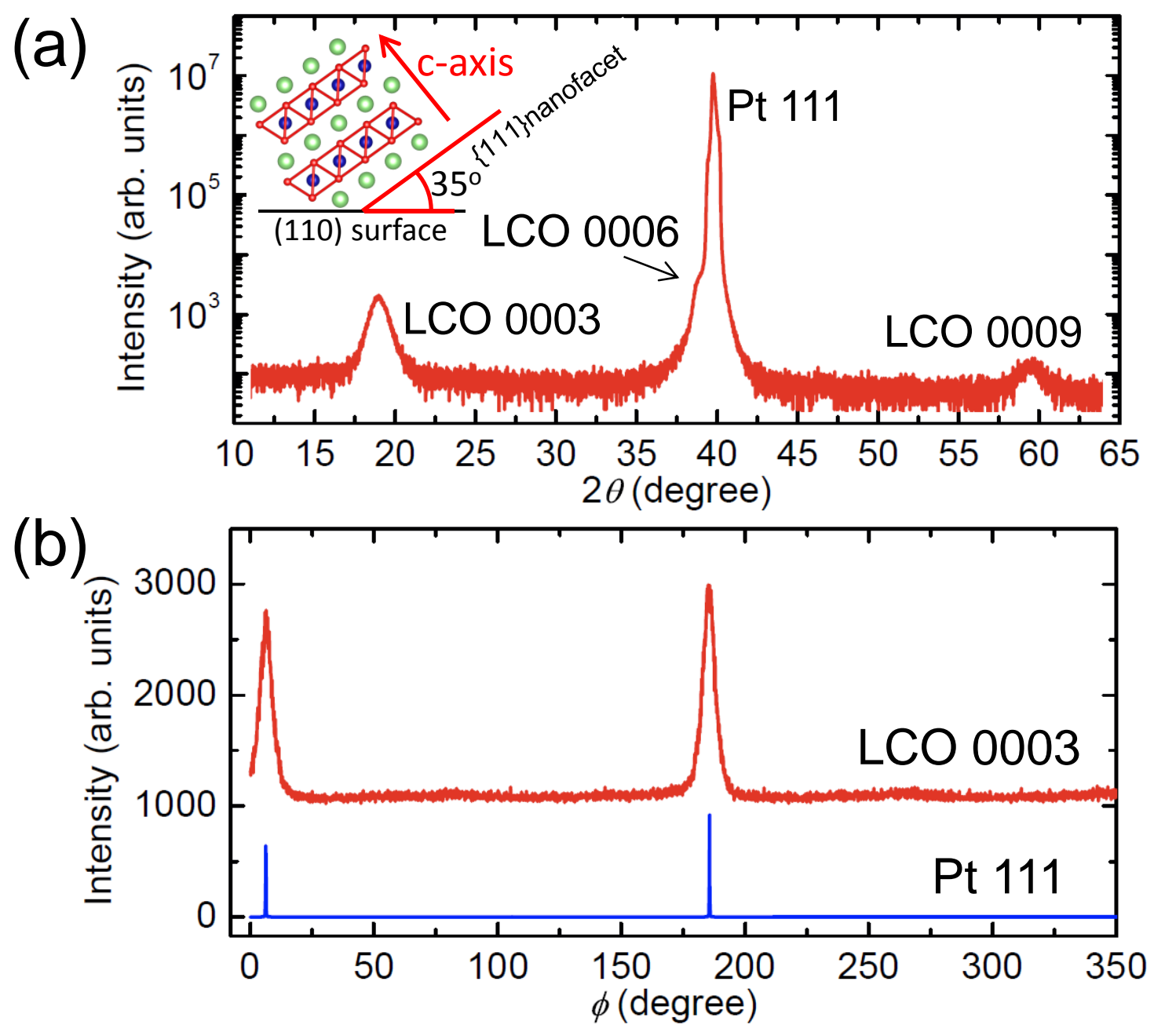

Fig. 2 XRD patterns of $\mathrm{LiCoO}_{2}$ thin films on a Pt(110) substrate etched with royal water. The inset shows the crystal alignment of $\mathrm{CoO}_{2}$ layers and substrate. (a) $\theta-2 \theta$ scan obtained at $\psi=35^{\circ}$. (b) $\phi$-scans of $\mathrm{LiCoO}_{2} 0003$ and Pt 111 diffractions obtained at $\psi=$ $35^{\circ}$. 


\section{(a) chemically-etched Pt(110) substrate}

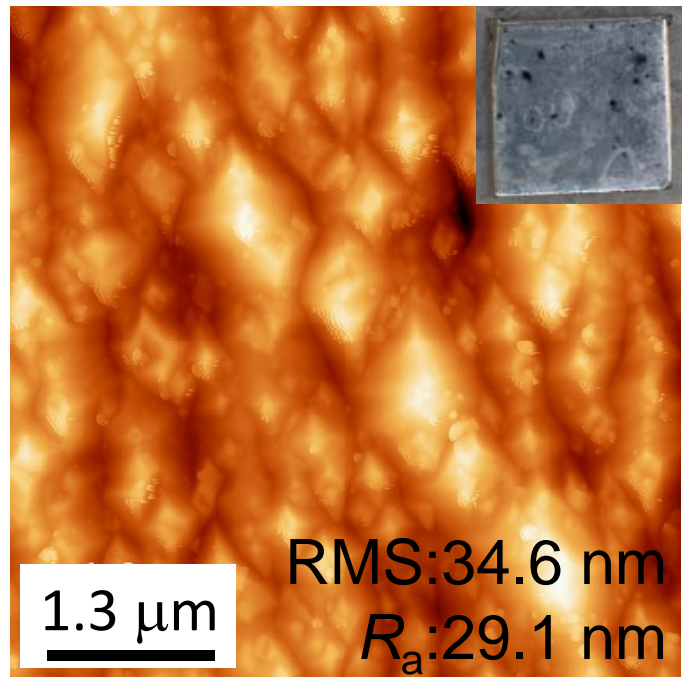

(b) reconstructed Pt(110) substrate

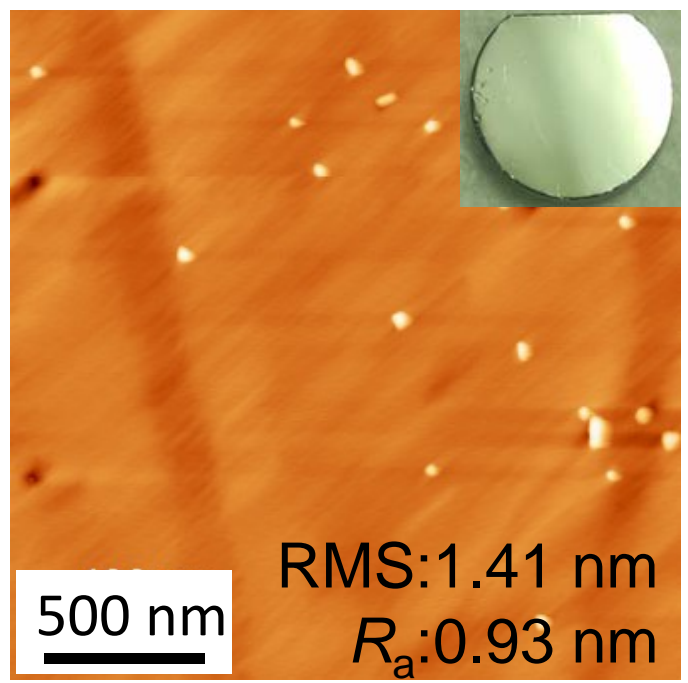

\section{(c)}

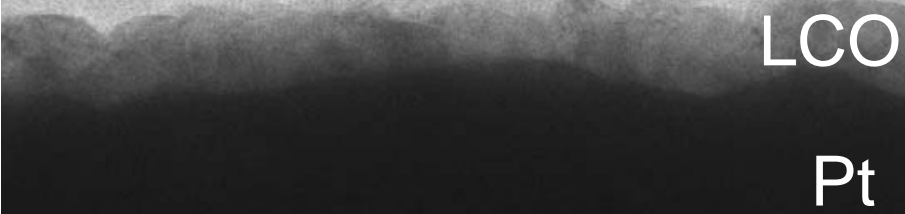

\section{$200 \mathrm{~nm}$}

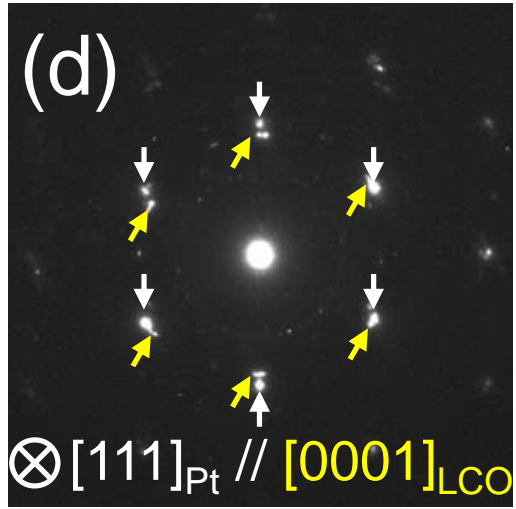

Fig. 3. AFM images of (a) etched and (b) reconstructed Pt(110) surfaces. The insets show photos of the Pt(110) substrates after the surface treatments. The chemically etched Pt(110) surface becomes lusterless after the etching treatments. (c) A TEM image of LCO on etched $\mathrm{Pt}(110)$. (d) An electron diffraction pattern taken from the region including $\mathrm{LCO}$ and Pt substrate. Electron incident axis is parallel to [111] of Pt substrate and [0001] and LCO. Sets of white and yellow arrows indicate the diffraction spots from Pt and LCO, respectively. 


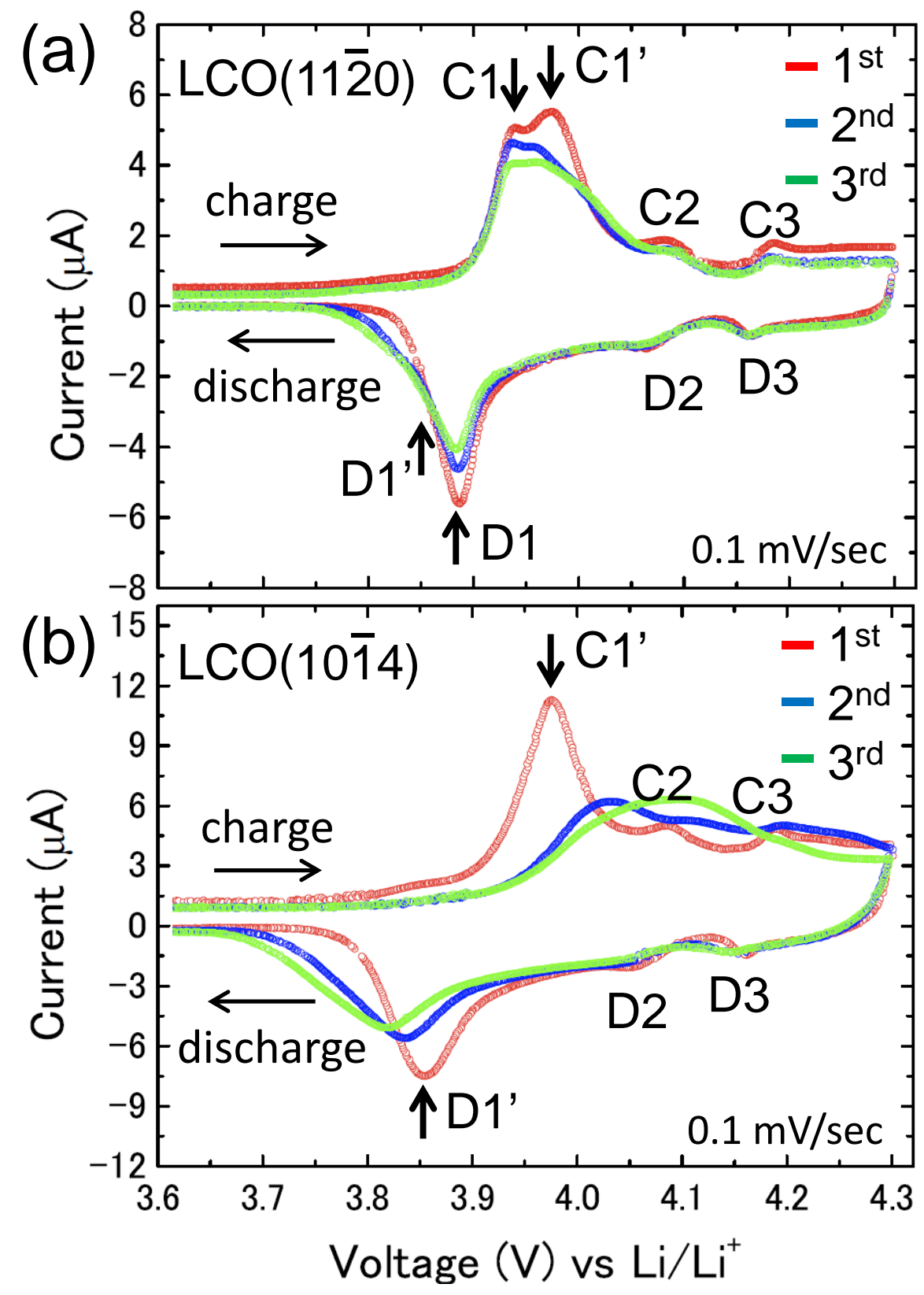

Fig. 4 Cyclic voltammogram of $\mathrm{LiCoO}_{2} / \mathrm{Pt}(110)$. (a) LCO(11-20) film on etched $\mathrm{Pt}(110)$. (b) $\mathrm{LCO}(10-14)$ film on reconstructed $\operatorname{Pt}(110)$. 